\title{
Reliability and validity of a Chinese version of the Career Decision-making Difficulties Questionnaire
}

\section{PETER A CREED* AND WONG OI YIN}

Griffith University, School of Psychology, PMB 50, Gold Coast Mail Centre, Gold Coast, Australia 9726 (*Author for correspondence: E-mail: p.creed@griffith.edu.au)

\begin{abstract}
This study tested the applicability of the 34-item CDDQ (Gati \& Saka, 2001) for use with adolescents from the Peoples Republic of China. The study devised a Chinese version of the scale using translation and back-translation, administered it to a sample of 514 Chinese adolescents, tested the psychometric properties of the scale in terms of factor structure and reliability, and examined its validity by testing associations with demographic variables (age, gender, paid work experience, socioeconomic status, and academic achievement) and scales tapping career decision-making self-efficacy and barriers. Results identified two stable factors for the CDDQ, namely Lack of Information and Inconsistent Information, but failed to confirm the third CDDQ factor of Readiness to make a career decision. Factor structure and reliability for the two identified factors were sound, and these factors related in the expected directions to demographic variables and the other career scales. Implications for use of the CDDQ in China are highlighted, and recommendations are made for the revision of the Readiness dimension.
\end{abstract}


For many young people, making a career decision can be a difficult and confusing task. They may lack a clear self-concept, have insufficient or conflicting information about the world of work, have fewer life experiences, limited interests and/or abilities, and might be affected by a number of interpersonal, social and environmental factors (Gati, Krausz, \& Osipow, 1996). Such confusion can result in a state of indecision, which can be exacerbated by problems associated with poor vocational identity, career barriers and inadequate decision-making skills (Osipow, 1999). Career indecision can have long-term negative consequences for young people, impacting on vocational life, well-being and their social situation (Mann, Harmoni, \& Power, 1989).

Reducing indecision can thus be an important issue for career decision-makers and those trying to assist, and diagnosing it can be an important first step in its resolution.

This need for diagnosis has led to the development of a number of psychometric tools that measure career indecision and its component parts. Indecision researchers have devised instruments that assess the sources of indecision, the difficulties associated with making career decisions (such as lack of relevant information and affective barriers) and developmental aspects associated with decision-making (Kelly \& Lee, 2002). However, there has been much criticism of these attempts, based on both theoretical and psychometric grounds (Kelly \& Lee, 2002; Tinsley, 1992). In response to this criticism, Gati, Krausz and Osipow (1996) proposed and empirically tested a decision theory based taxonomy of difficulties in career decision-making. The result of this, and other follow-up studies, was the development of a 44-item diagnostic instrument, the Career Decision-making Difficulties Questionnaire (CDDQ), which could be used both in research and applied settings (Osipow \& Gati, 1998; Gati, Osipow, Krausz, \& Saka, 2000; Gati \& Saka, 2001).

The taxonomy proposed by Gati et al. (1996) included three major domains of difficulties, namely Lack of Readiness, Lack of Information and Inconsistent Information, which were further divided into ten specific difficulty categories. Lack of Readiness included three categories of difficulties that could occur before engaging in the career decision-making process. These were lack of motivation, general indecisiveness, and dysfunctional beliefs. The other two domains contained categories of difficulties that could arise during the decision-making process. Lack of Information included four categories: lack of knowledge about the decision-making process, and lack of information about the self, career alternatives and where to obtain further career information. Inconsistent Information included three categories: unreliable information, internal conflicts and external conflicts.

Despite the multidimensional nature of the CDDQ and its theoretical base, it has been criticized on a number of grounds, for example, because of its narrow focus on the cognitive 
aspects of career decision-making (e.g., to the exclusion of affective and identity components) and for the poor psychometric properties of the Lack of Readiness subscales (lack of motivation, general indecisiveness, dysfunctional beliefs; Kelly \& Lee, 2002). Evidence for validity of the 44-item CDDQ has been shown by a number of studies (Lancaster, Rudolph, Perkins, \& Patten, 1999; Osipow \& Gati, 1998), although the Lack of Readiness subscales have consistently produced poor internal reliability coefficients (Albion \& Fogarty, 2002; Gati et al., 1996; Gati et al., 2000; Lancaster et al., 1999). Gati and Saka (2001) also produced a shortened and modified version of the CDDQ by reducing the 44 -item original version to 34 items (32 CDDQ items, and two items included as a validity check). These items were all drawn from the original CDDQ, and were adapted to be suitable for use with high school students.

Cultural factors are important contextual determinants when making career-related decisions (Lent, Brown, \& Hackett, 1996). People in Western cultures tend to be more individually oriented, with a focus on personal rights, well-being of the self and immediate family, and personal autonomy and accomplishments. In contrast, Chinese collectivism culture emphasizes group conformity and mutual obligations, where community goals and ethics are placed above personal goals and competencies (Oyserman, Coon, \& Kemmelmeier, 2002). Importantly, decision-making styles differ between Western and Chinese cultures. In the West, reasoning and attributions of causality are person- rather than situation- or group-orientated; whereas in the East, social conformity and collective decision-making predominates. Thus, parental and family expectations are more salient factors in the career decision-making process in China (Mau, 2000; Oyserman et al., 2002).

These cultural differences draw into question the suitability of using non-modified Western devised psychological tools with Chinese adolescents. Although there have been many reports examining the suitability of the CDDQ with Western college samples (Gati et al., 1996; Gati et al., 2000; Mau, 2000; Osipow \& Gati, 1998), and one study has tested the CDDQ with a Taiwanese sample (Mau, 2000), no studies to date have tested its suitability for use in the Peoples Republic of China. It is important to note that there are important historical and cultural differences between Taiwanese and Chinese cultures. Taiwan has experienced greater exposure to the West than China (Hartzell, 1988), which is reflected in adolescents' career focus, with, for example, Taiwanese students being found to be more aggressive in their career pursuits than Chinese students (Ihle, Sodowsky, \& Kwan, 1996).

The current study will contribute to the literature by testing the applicability of the 34-item CDDQ (Gati \& Saka, 2001) for use with adolescents from the Peoples Republic of China. The goals of the study were (a) to test the psychometric properties of a Chinese version of the CDDQ 
(in terms of factor structure and reliability), and (b) examine its validity by testing its relationship with demographic variables (age, gender, paid work experience, socioeconomic status and academic achievement) and two other career constructs that have been previously tested with adolescents in China, namely career decision-making self-efficacy (Hampton, 1999) and career barriers (Tien, 1998). It is particularly suitable to test the relationship between the CDDQ and self-efficacy and barriers, as both of these constructs are important career-related variables that have been shown to be associated with career difficulties in previous studies. Career decision-making self-efficacy has been found to be negatively associated with career decision-difficulties (Albion \& Fogarty, 2002; Mau, 2000) and perceptions of career barriers has been shown to be positively associated to career indecision and decision-making difficulties (Patton, Creed, \& Watson, 2003).

\section{Method}

\section{Participants}

Participants were 514 final year high school students attending the participating school at Nanghai, China. These students were chosen as they would be contemplating career decisions related to completing their secondary education. Seventy-five students were dropped from the analyses, primarily because list-wise deletion indicated the students had failed to answer all questions, although some cases were also removed when students failed to complete the survey satisfactorily (e.g., the student had responded by ticking all " $5 \mathrm{~s}$ " throughout the survey), and other cases were removed as they were extreme multivariate outliers. This left 439 students in the sample, which comprised 215 females (49\%), 216 males (49\%), and eight students (2\%) who did not indicate gender. The students had a mean age of 18.38 years $(S D=0.56$; Range $=18-21)$. A three-level measure of socioeconomic level was created using parental education and occupational status (Anderson \& Vervoorn, 1983). One hundred and eighty-five students (42\%) reported parents having primary school education, 238 (54\%) having parents with a high school education, and 11 students (3\%) having parents with higher education. Five students (1\%) did not respond to this question. Students provided a measure of their current academic achievement level. Forty-five students (10\%) indicated their current achievement level was "well below average", 116 (26\%) indicated that it was "below average", 230 (52\%) indicated "average", and 43 (10\%) indicated "above average". Five students (1\%) did not answer this question. Finally, students were asked to indicate their employment history. Only one student indicated that he had 
current paid part-time employment, which is typical of students in China, while 218 students $(50 \%)$ indicated some previous paid work experience, which was mostly vacation work.

\section{Instruments}

Career Decision-making Difficulties Questionnaire - Revised (CDDQ-R: Gati \& Saka, 2001). Students completed the 34-item Chinese translated version of the CDDQ-R, which comprised 32 CDDQ-R items, and two validity items that are not used in the scoring. Students were asked to indicate their level of agreement to each statement (sample item: "I find it difficult to make a career decision because I do not know what factors to take into consideration") on a 9-point scale, with endpoints of does not describe me and does describe me. Higher scores indicated more career decision-making difficulties.

The original CDDQ (Gati et al., 1996) contained 44 items and was designed to assess career decision-making difficulties across three subscales of Lack of Readiness, Lack of Information and Inconsistent Information related to the decision-making process. A Total difficulties score could also be obtained by summing scores across all domains. Gati et al. (1996) tested initial convergent, divergent and concurrent validity and found it to be satisfactory, and assessed multidimensionality using cluster analysis. These authors reported sound internal reliability scores for the Lack of Information (.95) and Inconsistent Information (.89) subscales, and for the Total scale (.94), but found low reliability for the Lack of Readiness subscale (.63). Albion and Fogarty (2002) and Lancaster et al. (1999) also reported low reliability for the Lack of Readiness subscale. Mau (2001) translated this 44 item version into Chinese and tested it on a sample of Taiwanese students. This author found a poor fit of the data to the original Gati et al. (1996) model when using structural equation modeling.

Gati and Saka (2001) reduced the 44 statements in the original CDDQ to 34, and adapted them for use with high school students. These changes were based on the results of item analyses, where highly correlating statements were combined or reduced to one, and irrelevant statements were deleted. Gati and Saka reported internal reliabilities for this shortened version that were consistent with the original scale, of .91 (Total), .62 (Lack of Readiness), .88 (Lack of Information) and .87 (Inconsistent Information). There have been no independent validation studies of the CDDQ-R, no studies have tested its factor structure using factor analysis or latent variable analysis, and no Chinese version was identified.

To create Chinese versions of the 34-item CDDQ and the other two scales used in the study: (a) three native Chinese speakers, who also spoke English, translated the measures into Chinese; 
(b) a monolingual Chinese speaker checked the readability of the translated versions, and (c) one native Chinese speaker, who also spoke English, then back translated them into English. The back-translated versions were compared with the original versions by the researchers for accuracy of meaning. Revisions were made to those items that were translated inaccurately. The final back-translated version of the CDDQ was sent to the originating author (Itamar Gati) for his approval for it to be administered (Itamar Gati, personal communication, July 2003).

Career Decision-making Self-efficacy Scale - Short Form (CDMSE-SF: Betz, Klein, \& Taylor, 1996). Students completed a 25-item Chinese translated version of the CDMSE-SF to assess confidence regarding ability to make career related decisions (sample item, "How confident are you that you could determine what your ideal job would be?"). Participants responded on a 5point scale, with end-points of no confidence at all and complete confidence. Higher scores indicated greater confidence. In developing the original CDMSE scale, Taylor and Betz (1983) included 10 behaviours indicative of each of the five career-choice competencies postulated by Crites (1971), of accurate self-appraisal, gathering occupational information, goal selection, making plans for the future, and problem solving. The CDMSE-SF contains five behaviours per competency. Betz et al. (1996) reported an internal reliability for the CDMSE-SF of .94, which is consistent with reliability coefficients reported in other studies (e.g., Creed, Patton, \& Watson, 2002). Two studies (Hampton, 1999; Mau, 2000) have used the CDMSE-SF with Chinese samples. The scale was found to be unidimensional when used with students from that country (Hampton) and to have internal consistencies ranging from .92 to .94. The internal reliability coefficient for the present sample was .87 .

Occupational Barriers Scale. Students completed a Chinese translated version of the Occupational Barriers Scale (Howell, Frese, \& Sollie, 1977, 1984). This scale asks respondents, "How much effect do you think each of the following things will have in keeping you from getting the job you desire?", for 10 barriers of "lack of interest by your parents, the school you are attending, not enough money to attend college or university, your not wanting to move, national shortage of 'good' jobs, local shortage of 'good' jobs, no college or university nearby, lack of information about existing opportunities, personal intelligence and salary". The original scale was modified to make it more suitable to Chinese students (e.g., "technical school and college" was replaced with "college and university", the item indicating race as a barrier was deleted, and the item asking about salary was included). Students were asked to indicate the level of effect on a 5-item response format with end-points of no effect and very much effect. Higher 
scores indicated more perceived barriers. Career-related barriers has been shown to be a meaningful construct for Chinese adolescents (Tien, 1998). The internal reliability coefficient for the scale was .71.

Demographic Questions. Students were asked to indicate their age, gender, academic achievement (5-point scale with end-points of well below average and above average), whether they had paid part-time or casual work experience, the highest education level of their father and mother (4-point scale of never received education/primary school/high school/university), and father's and mother's current employment situation.

\section{Procedure}

The survey forms, containing the three translated scales (Career Decision-making Difficulties Questionnaire, Career Decision-making Self-efficacy, Occupational Barriers) and the demographic questions, were administered to all volunteering students in the one grade in the secondary school that participated in the study. The classroom teachers, who had been provided with instructions regarding the administration protocol, administered the survey forms. A written group careers audit was provided to the school following the data collection.

\section{Data Analysis}

To test the dimensionality of the Career Decision-making Difficulties Questionnaire, the sample of 439 students was randomly split into two separate groups. The groups were first compared to determine if any bias resulted from the split. One resultant group was then subjected to an exploratory factor analysis (principal-axis factor analysis with direct oblimin rotation) and the second group was subjected to a confirmatory factor analysis (Arbuckle \& Wothke, 1995). To test the validity of the Career Decision-making Difficulties Questionnaire, a series of crosssectional analyses was then conducted using the Career Decision-making Self-efficacy Scale, the Occupational Barriers Scale and the demographic questions. 


\section{Results}

\section{Random Split}

The full data set was randomly split into two samples, indicated as Sample A and Sample B. In order to test for bias in this split, the two samples were compared on demographic variables, the total scores of the Career Decision-making Difficulties Questionnaire, the Career Decisionmaking Self-efficacy Scale and the Occupational Barriers Scale. These results indicated that Samples A and B did not differ on age, gender, socioeconomic level, level of academic achievement, whether students had work experience, the Career Decision-making Difficulties Questionnaire, the Career Decision-making Self-efficacy Scale, and the Occupational Barriers Scale. These results indicate that no bias was identified as a result of splitting the full database, and that the two split samples could be considered representative of the original. Summary data for Samples A, B and the Total Sample are reported in Table 1. 
Table 1. Summary data for Samples A, B and Total Sample on the Career Decision-making Difficulties Questionnaire, the Career Decision-making Self-efficacy Scale and the Occupational Barriers Scale

\begin{tabular}{|c|c|c|c|c|c|c|c|c|c|}
\hline \multirow[b]{2}{*}{ Variable } & \multicolumn{3}{|c|}{ Sample A } & \multicolumn{3}{|c|}{ Sample B } & \multicolumn{3}{|c|}{ Total } \\
\hline & $N$ & $M$ & $S D$ & $N$ & $M$ & $S D$ & $N$ & $M$ & $S D$ \\
\hline & & & & & & & & 153.53 & 37.82 \\
\hline CDDQ & 219 & 154.96 & 40.26 & 220 & 152.10 & 35.26 & 439 & & \\
\hline CDMSE-SF & 219 & 82.03 & 12.48 & 220 & 81.63 & 12.31 & 439 & 81.83 & 12.38 \\
\hline OBS & 212 & 28.03 & 6.21 & 216 & 27.94 & 6.34 & 428 & 27.98 & 6.27 \\
\hline
\end{tabular}

\footnotetext{
Note. Sample sizes vary as not all students completed all scales; CDDQ = Career Decision-making Difficulties Questionnaire; CDMSE-SF = Career Decision-making Selfefficacy Scale; OBS = Occupational Barriers Scale
} 
Factor Structure of the Career Decision-making Difficulties Questionnaire

To estimate the factor structure of the CDDQ, an exploratory factor analysis, using principal axis factoring with an oblique (direct oblimin) rotation, was conducted on Sample A. Initial results, using all 32 items from the CDDQ (the two validity items were not included), indicated 9 factors with eigenvalues $>1$ to be rotated. When this was done, the rotated factor structure was factorially complex, with multiple cross-loadings and several factors with only one or two items that loaded greater than .40 , the size of loading required to be significant at $p<.05$ for a sample size of 200 (Hair, Anderson, Tatham, \& Black, 1995). The Scree plot indicated that three factors should be rotated. When this was done, the rotated factor structure was still factorially complex with nine items not loading on any factors and the smallest factor having only three significant item loadings. Following recommendations by Hair et al. (1995), two and four factors were also rotated. When four factors were rotated, the two smallest factors each contained three significantly loading items. When two factors were rotated, two strong factors emerged, although there were two cross-loadings, two items that loaded on a factor different to what was originally intended, and 9 items that did not load on either factor. This was the best solution identified.

When the cross-loading, misplaced, and non-loading items were removed, these two factors accounted for $46.91 \%$ of the variance. This solution was factorially simple and interpretable, with 11 items loading on Factor 1 (eigenvalue $=6.75$, variance explained $=32.74 \%$ ) and eight items loading on Factor 2 (eigenvalue $=2.16$, variance explained 11.39\%). The correlation between the two factors was $r=.37$. The internal reliability coefficient for Factor 1 was .90 and for Factor 2 was .74. The 11 items loading on Factor 1 were all from the original 12-item CDDQ Lack of Information subscale. The eight items that loaded on Factor 2 were all from the original 10-item CDDQ Inconsistent Information category. As no items from the original 10-item Readiness subscale formed a discrete factor, corrected item-total correlations for this subscale were examined. These ranged from -.09 to .28, indicating poor relationships between the individual items in the Readiness subscale and the Readiness subscale total. The internal reliability coefficient for this Readiness subscale was .29; further indicating poor construct validity for this subscale. Factor loadings resulting from the above analysis are reported in Table 2. 
Table 2. Principal axis factor estimates of oblique (direct oblimin) factor loadings for the 19-item Chinese translation of the CDDQ (Random Sample A, $N=219$ )

Items (in some cases abbreviated)

F1 F2

I find it difficult to make a career decision because...

19. I do not know what my abilities and/or personality traits will be like in the $\begin{array}{llll}\mathbf{8 2} & -.09\end{array}$ future

14. I do not know what factors to take into consideration $\quad \begin{array}{cc}\mathbf{7 6} & -.04\end{array}$

16. I still do not know which occupations interest me $\quad \begin{array}{ccc}.74 & -.23\end{array}$

18. I do not have enough information about my competencies and/or about $\quad \begin{array}{lll}\mathbf{. 7 3} & .01\end{array}$ my personality traits

17. I am not sure about my career preferences yet $\quad \begin{array}{lll}.71 & -.06\end{array}$

13. I do not know what steps I have to take $\quad \begin{array}{cc}\mathbf{7 0} & .02\end{array}$

15. I do not know how to combine the information I have about myself with $\quad \begin{array}{cc}.67 & .16\end{array}$ the information I have about the different careers

23. I do not know what careers will look like in the future $\quad .65$

21. I do not have enough information about the characteristics of the $\quad \begin{array}{rr}\mathbf{6 3} & .07\end{array}$ occupations and/or training programs that interest me

20. I do not have enough information about the variety of occupations or $\quad \begin{array}{rr}\mathbf{5 9} & .15\end{array}$ training programs that exist

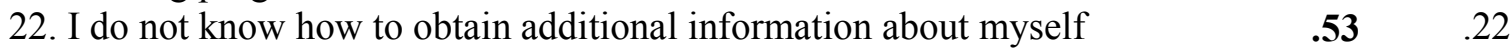

34. There are contradictions between the recommendations made by different $\quad \begin{array}{lll}-.07 & \mathbf{. 6 8}\end{array}$ people who are important to me about the career that suits me

33. People who are important to me do not agree with the career options I am $\quad \begin{array}{lll}-.13 & \mathbf{. 5 9}\end{array}$ considering and/or the career characteristics I desire

28.I m equally attracted to a number of careers and it is difficult for me to $\quad \begin{array}{ccc}\mathbf{4} & -.049\end{array}$ choose among them

26. I have contradictory data about my abilities and/or personality traits $\quad .23 \quad \mathbf{. 4 2}$

27.I have contradictory data about the existence or the characteristics of a $\quad .27 \quad \mathbf{. 4 1}$ particular occupation or training program

25. I constantly change my career preferences $\quad \begin{array}{ll}\mathbf{. 4 1} \\ \text { 21. }\end{array}$

31. my preferences can not be combined in one career, and I do not want to $\quad .12 \quad \mathbf{. 4 0}$ give any of them up

29. I do not like any of the occupation or training programs to which I can be $\quad \begin{array}{lll}.02 & \mathbf{3 6}\end{array}$ admitted

Note. Kaiser-Meyer-Olkin measure of sampling adequacy $=.88$; Bartlett's Test of Sphericity is $\chi^{2}(171)=1819.59 ; p$ $<.001$

To confirm the factor structure of the Chinese version of the CDDQ, a confirmatory factor analysis (CFA), based on the 220 students in Sample B, was estimated utilizing maximum likelihood procedures using AMOS Version 4.0 (Arbuckle \& Wothke, 1995). In a CFA, an a priori structure is posited and the adequacy of how well the data fits this structure is tested. For this analysis, two models were tested. In the first model, each cluster of CDDQ items that was identified in the exploratory factor analysis in the present study to represent a factor was allowed to load freely on a latent factor. Thus, items 13-23 were allowed to load freely on a single latent 
factor of Lack of Information, and items 25-29, 31 and 33-34 were allowed to load freely on a single latent factor of Inconsistent Information. In the second model, the original 32 items of the CDDQ were allowed to load on the three original hypothesized factors. Items 1-7 and 9-11 were allowed to load on the Readiness latent factor; items 13-24 were allowed to load on the Lack of Information latent factor; and items 25-34 were allowed to load on the Inconsistent Information latent factor. The correlations between the two factors in Model 1 and among the three factors in Model 2 were freely estimated. Variances for all latent factors were fixed at unity to identify the models.

The results of the CFA analyses indicated that Model 1, the two-factor model, was a better fit to the data than Model 2, the original three-factor model. Secondly, the results of almost all of the goodness of fit indices, which indicate the adequacy of a model (Byrne, 2001), indicated that Model 1 was a good fit to the data, while the fit for Model 2 was less than acceptable (see Table 3). Further, for Model 1, all factor loadings were significant $(p<.05)$, whereas for Model 2 , factor loadings for Items 2, 7 and 9 on Factor 1 (Readiness) were non-significant. Post-hoc modifications, in the form of allowing error terms to correlate, were made to both models to enable the best fit to be determined for both. In Model 1 (two factor), correlated error terms were allowed for item pairs 16-17, 18-19, 18-23, 18-21, 20-21, 21-22, 26-26 and 33-34. In Model 2 (three factor), correlated error terms were allowed for item pairs 1-10, 2-3, 9-11, 15-17, 16-17, 18-19, 18-21, 18-23, 20-21, 21-22, 26-27 and 33-34. When similar response formats are utilized for survey items, correlated error terms can be used to correct for this method effect. Marsh and Grayson (1995) indicated "method effect refers to the influence of a particular method that inflates a correlation among the different traits measured with the same method" (p. 177). Jöreskog and Sörbom (1996) indicate that where measures are repeated, as in a scale, "there is a tendency for the measurement errors... to correlate over time due to memory or other retest effects" (p. 222). Subjective indices for these analyses are reported in Table 3. Summary data for the two factors are reported in Table 4. 
Table 3. Chi-square and goodness-of-fit indices for Chinese version of CCDQ (Random Sample $\mathrm{B}, \mathrm{N}=220)$

\begin{tabular}{lllllllllll}
\hline Model & $d f$ & $\chi^{2}$ & CMIN/DF & GFI & AGFI & IFI & TLI & CFI & RMSEA & PCLOSE \\
\hline 2-Factor & 143 & $244.62 * * *$ & 1.71 & .90 & .86 & .94 & .92 & .94 & .06 & .17 \\
3-Factor & 449 & $671.14 * * *$ & 1.50 & .84 & .81 & .90 & .88 & .89 & .05 & .70
\end{tabular}

Note. GFI = Goodness of Fit, AGFI = Adjusted Goodness of Fit, IFI = Incremental Fit Index (Hair, Anderson, Tatham, \& Black, 1995), TLI - Tucker-Lewis Index (Tucker \& Lewis, 1973), CFI = Comparative Fit Index (Bentler, 1990), RMSEA = Root Mean Square Error of Approximation, PCLOSE = Probability of Close Fit (Browne \& Cudeck, 1993), *** $=p<.001$.

Table 4. Summary data for Random Samples A, B and Total Sample on CDDQ Factors 1 and 2

\begin{tabular}{lccccccccc}
\hline & \multicolumn{3}{c}{ Sample A } & \multicolumn{3}{c}{ Sample B } & \multicolumn{3}{c}{ Total } \\
\cline { 2 - 10 } Factor & $N$ & $M$ & $S D$ & $N$ & $M$ & $S D$ & $N$ & $M$ & $S D$ \\
\hline CDDQ (F1) & 219 & 53.47 & 21.76 & 220 & 52.05 & 20.02 & 439 & 52.76 & 20.90 \\
CDDQ (F2) & 219 & 32.25 & 12.32 & 220 & 31.39 & 11.69 & 439 & 31.82 & 12.00 \\
\hline
\end{tabular}

To further test the construct validity of the Chinese version of the CDDQ, all 54 items from the four scales in the study (CDDQ - Lack of Information factor $=11$ items, CDDQ Inconsistent Information factor $=8$ items, Career Decision-making Self-efficacy scale $=25$ items, Occupational Barriers Scale $=10$ items) were included in a principal axis factor analysis, utilizing a direct oblimin rotation, and using the full sample of 428 students. The Kaiser-MeyerOlkin Measure of Sampling Adequacy (.87) and Bartlett's Test of Sphericity $\left(\chi^{2}=7901.72, d f=\right.$ $1431, p<.001$ ) indicated the suitability of the data for factor analysis (Tabachnik \& Fidell, 1996). Four factors were rotated (to reflect the four scales included). This solution was factorially simple and interpretable, with all items loading on their appropriate factors. All four factors had eigenvalues greater than one, and the solution accounted for $34.37 \%$ of the variance. There were no substantive cross-loadings, one Career Decision-making Self-efficacy item had a weak loading on its factor, and several Occupational Barriers Scale items had weak loadings on their factor. This analysis demonstrated the independence of the two CDDQ subscales from other career related variables. Summary data for this analysis are reported in Table 5. 
Table 5. Eigenvalues, percentage of variance explained and inter-factor correlations for the CDDQ - Lack of Information, CDDQ - Inconsistent Information, Career Decision-making Selfefficacy Scale, and Occupational Barriers Scale (Full sample, $N=428$ )

\begin{tabular}{lcccccc}
\hline \multicolumn{1}{c}{ Scale } & Eigenvalue & $\begin{array}{c}\text { Variance } \\
\text { Explained }\end{array}$ & & \multicolumn{3}{c}{ Correlations } \\
& & & 1 & 2 & 3 & 4 \\
\hline CDDQ (F1) & 10.09 & 18.69 & - & .34 & -.23 & .26 \\
CDDQ (F2) & 3.79 & 7.01 & & - & -.20 & .14 \\
CDMSE & 2.53 & 4.69 & & & - & -.19 \\
OBS & 2.14 & 3.97 & & & - \\
\hline
\end{tabular}

Note. CDDQ = Career Decision-making Difficulties Questionnaire; CDMSE = Career Decision-making Selfefficacy Scale; OBS = Occupational Barriers Scale

Validity of the Career Decision-making Difficulties Questionnaire

Concurrent validity was tested by examining the associations between the CDDQ - Lack of Information, the CDDQ - Inconsistent Information, the Career Decision-making Self-efficacy Scale and the Occupational Barriers Scale (Table 5). All associations were in the expected direction. Lack of Information and Inconsistent Information were moderately associated, with students who reported a lack of information also reporting receiving inconsistent information. Lack of Information had moderate associations with Career Decision-making Self-efficacy and the Occupational Barriers Scale, with students who reported a lack of information also reporting lower self-efficacy and more perceived barriers. Inconsistent Information was moderately associated with Career Decision-making Self-efficacy and weakly associated with the Occupational Barriers Scale, with students who reported receiving inconsistent information also reporting lower self-efficacy and more perceived barriers. These results give confidence that the two subscales are related to one another and the other career variables in the manner expected (Gregory, 2000)

Construct validity for the two factors was further examined by testing for expected differences in subgroups identified by the demographic questions used in this study (Gregory, 2000). Students were questioned on their gender, paid work experience, socioeconomic status and academic achievement. Students were expected to perceive fewer career difficulties if they were male, had higher socioeconomic status, had higher academic achievement, and had paid work experience (Creed \& Patton, 2003; Patton \& Creed, 2001). Each of these variables was 
addressed individually using ANOVAs. Compared with females, males reported significantly lower Lack of Information, $F(1,429)=4.61, p<.05$, and Inconsistent Information scores, $F(1$, $429)=4.00, p<.05$. There were no differences based on socioeconomic level. Differences were found for level of academic achievement for Inconsistent Information, $F(3,430)=3.80, p<.01$, and Lack of Information, $F(3,430)=3.37, p<.05$. Differences were in the expected direction, with "below average" ( $p<.01)$ and "average" $(p<.05)$ students reporting higher difficulty scores than "above average" students. Also, "below average" students $(p<.05)$ reported higher difficulty scores than "above average" students. Lastly, no differences were identified based on previous work experience.

Thus, support for the content and construct validity of the two factors of the Chinese translation of the CDDQ - Inconsistent Information and the CDDQ - Lack of Information was indicated in two ways: (a) by the subscales relating to external variables of gender and achievement in the expected directions, and (b) by the factor analyses reported earlier (Gregory, 2000). The subscales were not related to age or employment, which may be explained by the narrow age range of the students and the fact that very few of the students reported paid employment. Further validity studies are required to confirm the usefulness of this Chinese version of the CDDQ.

\section{Discussion}

The current study successfully translated the 34-item version of the CDDQ from English into Chinese. Examination of the factor structure of this Chinese version indicated that only two factors were defined, rather than three, as was intended in the original English version of the scale. The two, largely uncorrelated, factors to emerge were Lack of Information (which contained 11 of the 12 original items for this subscale) and Inconsistent Information (containing 8 of the 10 original items from this subscale). Despite these factors/subscales being shorter than the original subscales by one and two items respectively, both can be considered to mirror the original subscales. The internal reliabilities for these two subscales were adequate, being $>.70$. No factor emerged for the Readiness subscale, and when the original Readiness items were tested separately, all corrected item-total correlations were weak $(<.28)$ and the internal reliability was unacceptable, at 29 .

These results are consistent with previous studies that have identified low internal reliability for the Readiness subscale (e.g., Albion \& Fogarty, 2002; Gati et al., 1996; Gati et al., 2000; Lancaster et al., 1999; Mau, 2001). More importantly, the results indicate that the CDDQ 
Readiness items are not sufficiently homogeneous to warrant their use with Chinese adolescents. It is not possible to determine from this study whether the poor results for the Readiness subscale reflect the poor psychometric properties for this subscale generally, perhaps exacerbated by the translation from English into Chinese, or whether this result reflects cultural differences, where readiness to make a career decision for the Chinese adolescents is linked to group norms and values and cannot be interpreted as an individually relevant act (Earley, 1994). Possible cultural explanations include (a) the economy of China, where many still rely on the government to take care of their career choice, and where many still expect to work in a government setting; (b) the labour market inexperience of the students in the study (only one student reported having paid part-time employment; a situation very different to Western high school adolescents); and (c) the life stage of the students, who were all in middle school, and who may have expectations of continuing their education rather than entering the workforce. There was some support for cultural effects, over and above the psychometric limitations of the scale, by Mau (2001), who found that the dependent style of Taiwanese students meant that their career decision-making tended to reflect familial and societal expectations, rather than the students' own desires.

Gati (1998) and Gati et al. (1996) indicated that certain subscales within the CDDQ are less refined than others. It is apparent that the Lack of Readiness subscale is in need of more development in this cross-cultural setting. This might require the development and testing of culturally appropriate items in relation to Readiness. However, future studies also need to examine the applicability of the readiness construct in collective cultures, such as China. This recommendation is important as the CDDQ has been proposed to differentiate two separate phases to career decision-making, one prior to the decision-making process, and a second during the decision-making process (Gati \& Saka, 2001; Gati et al., 1996).

In relation to validity, the two CDDQ subscales of Lack of Information and Inconsistent Information were shown to have good construct validity from the initial factor analyses, and shown to have good divergent validity when factor analysed with the other scales used in the study, namely the Career Decision-making Self-efficacy scale and the Occupational Barriers scale. Correlations between the Lack of Information and Inconsistent Information subscales and the Career Decision-making Self-efficacy scale and the Occupational Barriers scale were in the expected direction, with career decision-making difficulties being negatively associated with efficacy and positively associated with barriers. Also consistent with expectations, males reported more career decision-making difficulties than females, and lower achievers reported more difficulties than higher achievers. Typically, males have been found to be less career mature than girls (Patton \& Creed, 2001), although this result might also represent cultural 
effects in a society where young women may still be compelled to select from a narrow range of gender-specific occupational possibilities (Herr \& Cramer, 1992), and young males have higher parent and societal expectations and are expected to be more career-orientated (Hoffman, 1977). Lastly here, it is to be expected that higher achieving students would present with fewer difficulties on a scale that is focused on the acquisition of information and being able to make sense of inconsistent information. To our knowledge, this result has not been reported elsewhere, but is not likely to be culturally specific.

The authors were not able to identify scales similar to the CDDQ that have been developed specifically for the Chinese culture, and there are no official Chinese translations of popular Western career assessment instruments, such as the Self-directed Search (Holland, 1994). This is a serious gap in the tools available to those in China who assist with career decisions. Simple translations are of course less desirable than tests developed specifically within a particular culture, as has been demonstrated in the current study (see Cheung, Cheung, Wada, \& Zhang, 2003). The CDDQ has been developed using sound theory and methodology. However, the current translation into Chinese has identified a serious weakness in relation to its use there. While further development and refinement of the CDDQ, especially the Readiness construct, will make it more suitable for the Chinese context, the underlying theoretical assumptions also need to be tested for their applicability in Asia. Having said this, we do acknowledge that this translation of the CDDQ was assessed on a narrow age group of students from the same school, and that a more representative sample of students would have been more desirable.

Notwithstanding this, the present study provides some support for the theoretical taxonomy of career decision-making difficulties proposed by Gati et al. (1996). The translated CDDQ may be used for needs assessments; for example, within school counselling, or it can be used to facilitate the identification of students who have difficulties related to the two identified factors Lack of Information and Inconsistent Information. The CDDQ also makes it possible to assess the outcomes of intervention aimed at reducing general or specific career decision-making difficulties. However, further research aimed at improving the scales' general reliability and validity is needed before the CDDQ can be introduced as a diagnostic instrument. Importantly, when using the CDDQ in a Chinese setting, some other means of assessing Lack of Readiness needs to be used.

Finally, Hesketh and Rounds (1995) argued that the main psychological dimension differentiating individuals across cultures on vocational grounds was individualism-collectivism, where Western work cultures value independence and Asian work cultures, such as in China and Hong Kong, value more collectivistic approaches. This means that careers counsellors in China 
have to help their clients achieve a balance between personal and group (i.e., family, work unit) goals and loyalties. The challenge here for researchers is to understand the role and influence of important others in the lead-up to career decision-making and in the decision-making process itself in China and incorporate this in any assessment devise. 


\section{References}

Anderson, D.S., \& Vervoorn, A.E. (1983). Access to privilege: Patterns of participation in Australian post-secondary education. Canberra, Australia: Australian National University Press.

Albion, M.J., \& Fogarty, G.J. (2002). Factors influencing career decision making in adolescents and Adults. Journal of Career Assessment, 10, 91-126.

Arbuckle, J.L. \& Wothke, W. (1995). Amos 4.0 User's Guide. Chicago: Small Waters Corporation.

Bentler, P.M. (1990). Comparative fit indexes in structural models. Psychological Bulletin, 107, 238-246.

Betz, N.E., Klein, K., \& Taylor, K.M. (1996). Evaluation of a short form of the Career DecisionMaking Self-Efficacy scale. Journal of Career Assessment, 4, 47-57.

Browne, M.W. \& Cudeck, R. (1993). Alternative ways of assessing model fit. In K.A. Bollen \& J.S. Long (Eds.). Testing structural equation models (pp. 445-455). Newbury Park, CA: Sage.

Byrne, B. (2001). Structural equation modeling with AMOS. London: Lawrence Erlbaum.

Cheung, F.M., Cheung, S.F., Wada, S., \& Zhang, J. (2003). Indigenous measures of personality assessment in Asian countries: A review. Psychological Assessment, 15, 280-289.

Creed, P.A., \& Patton, W. (2003). Differences in career attitude and career knowledge for high school students with and without paid work experience. International Journal for Educational and Vocational Guidance, 3, 21-33.

Creed, P.A., Patton, W., \& Watson, M. (2002). Cross-cultural equivalence of the Career Decision-making Self-efficacy scale - Short Form: An Australian and South African comparison. Journal of Career Assessment, 10, 327-342.

Crites, J. (1971). The maturity of vocational attitudes in adolescence. Washington, DC: APGA.

Earley, P.C. (1994). Self or Group? Effects of Training on Self-Efficacy and Performance. Administrative Science Quarterly, 39, 89-117.

Gati, I. (1998). Using career-related aspects to elicit preferences and characterize occupations for a better person-environment fit. Journal of Vocational Behavior, 52, 345-356.

Gati, I., Krausz, M., \& Osipow, S.H. (1996). A taxonomy of difficulties in career decisionmaking. Journal of Counseling Psychology, 43, 510-526. 
Gati, I., Osipow, S.H., Krausz, M., \& Saka, N. (2000). Validity of the career decision-making difficulties questionnaire: Counselees' versus career counselors' perceptions. Journal of Vocational Behavior, 56, 99-113.

Gati, I., \& Saka, N. (2001). Career-related decision-making difficulties of high-school students. Journal of Counseling and Development, 79, 331-340.

Gregory, R.J. (2000). Psychological testing: History, principles and applications ( $3^{\text {rd }}$ ed.). Boston: Allyn \& Bacon.

Hair, J., Anderson, R., Tatham, R., \& Black, W. (1995). Multivariate data analysis. (4 ${ }^{\text {th }}$ ed.). New Jersey: Prentice Hall.

Hampton, N.Z. (1999). Psychometric properties of the Career Decision-Making Self-Efficacy Scale in Chinese college students. Paper presented at the Annual Convention of the American Psychological Association, Boston, MA.

Hartzell, R.W. (1988). Harmony in conflict: Active adaptation to life in present-day Chinese society. Taipei, Taiwan: Caves.

Herr, E.L., \& Cramer, S.H. (1992). Career guidance and counseling through the lifespan: Systematic approaches. New York: Harper Collins.

Hesketh, B., \& Rounds, J. (1995). International cross-cultural approaches to career development. In W.B. Walsh \& S.H. Osipow, Handbook of vocational psychology: Theory, research, and practice ( $2^{\text {nd }}$ ed., pp. 367-390). Mahwah, NJ: Erlbaum.

Hoffman, L.W. (1977). Changes in family roles, socialization and sex differences. American Psychologist, 32, 644-657.

Holland, J.L. (1994). The Self-Directed Search: Professional manual. Odessa, FL: Psychological Assessment Resources.

Howell, F.M., Frese, W., \& Sollie, C.R. (1977). Ginzberg's theory of occupational choice: A reanalysis of increasing realism. Journal of Vocational Behavior, 11, 332-346.

Howell, F.M., Frese, W., \& Sollie, C.R. (1984). The measurement of perceived opportunity for occupational attainment. Journal of Vocational Behavior, 25, 325-346.

Ihle, G.M., Sodowsky, G.R., \& Kwan, K.L. (1996). Worldviews of women: Comparisons between White American clients, White American counselors, and Chinese international students. Journal of Counseling and Development, 74, 300-306.

Jöreskog, K.G., \& Sörbom, D. (1996). LISREL8: User's reference guide. Chicago: Scientific Software International.

Kelly, K.R., \& Lee, W. (2002). Mapping the domain of career decision problems. Journal of Vocational Behavior, 61, 302-326. 
Lancaster, P.L., Rudolph, C., Perkins, S., \& Patten, T. (1999). Difficulties in career decision making: A study of the reliability and validity of the Career Decision Difficulties Questionnaire. Journal of Career Assessment, 7, 393-413.

Lent, R.W., Brown, S.D., \& Hackett, G. (1996). Career development from a sociocognitive perspective. In D. Brown \& L. Brooks (Eds.), Career choice and development ( $3^{\text {rd }}$ ed., pp. 373-422). San Francisco: Jossey-Bass.

Mann, L., Harmoni, R., \& Power, C. (1989). Adolescent career decision making: The development of competence. Journal of Adolescence, 12, 265-278.

Mau, W.C. (2000). Cultural differences in career decision-making styles and self-efficacy. Journal of Vocational Behavior, 57, 365-378.

Mau, W.C. (2001). Assessing career decision-making difficulties: A cross-cultural study. Journal of Career Assessment, 9, 353-364.

Marsh, H.W., \& Grayson, D. (1995). Latent-variable models of multitrait-multimethod data. In R.H. Hoyle (Ed.). Structural equation modeling: Issues and applications (pp. 177-198). Newbury, CA: Sage.

Osipow, S.H. (1999). Assessing career indecision. Journal of Vocational Behavior, 55, 147-154.

Osipow, S.H., \& Gati, I. (1998). Construct and concurrent validity of the career decision-making difficulties questionnaire. Journal of Career Assessment, 6, 347-364.

Oyserman, D., Coon, H.M., \& Kemmelmeier, M. (2002). Rethinking individualism and collectivism: Evaluation of theoretical assumptions and meta-analyses. Psychological Bulletin, 128, 3-72.

Patton, W., \& Creed, P.A. (2001). Developmental issues in career maturity and career decision status. The Career Development Quarterly, 49, 336-351.

Patton, W., Creed, P.A., \& Watson, M. (2003). Perceived work related and non-work related barriers in the career development of Australian and South African adolescents. Australian Journal of Psychology, 55, 74-82.

Tabachnik, B.G., \& Fidell, L.S. (1996). Using multivariate statistics. New York: Harper-Collins College Publishers.

Taylor, K.M., \& Betz, N.E. (1983). Applications of self-efficacy theory to the understanding and treatment of career indecision. Journal of Vocational Behavior, 22, 63-81.

Tien, H.S. (1998). Development and initial validation of a Chinese career barriers inventory. Paper presented at the Annual Convention of the American Psychological Association, San Francisco. 
Tucker, L.R., \& Lewis, C. (1973). A reliability coefficient for maximum likelihood factor analysis. Psychometrika, 38, 1-10. 